\title{
ChemComm
}

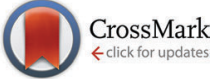

Cite this: Chem. Commun., 2016, 52, 1964

Received 28th October 2015 Accepted 8th December 2015

DOI: $10.1039 / c 5 c c 08968 a$

www.rsc.org/chemcomm

\section{Supramolecular surface adhesion mediated by azobenzene polymer brushes $\dagger$}

\author{
Oliver Roling, $\$$ Lucas Stricker $\$$ Jens Voskuhl,$\$$ Sebastian Lamping and \\ Bart Jan Ravoo*
}

\begin{abstract}
Surface immobilised polymer brushes containing azobenzene units were prepared using a combination of microcontact chemistry and surface-initiated atom transfer radical polymerisation (SI-ATRP). These brushes were investigated using AFM, XPS and UV/vis spectroscopy. It was shown that two surfaces bearing azobenzene brushes can be glued together in the presence of a $\beta$-cyclodextrin polymer and hold as much as $700 \pm 150 \mathrm{~g} \mathrm{~cm}^{-2}$.
\end{abstract}

The use of supramolecular structures in materials science is both a highly challenging and a very promising strategy since it is necessary to use an orchestration of multiple weak non-covalent interactions to form a stable yet stimulus responsive material. To date several approaches were developed to use weak supramolecular interactions such as van der Waals, hydrophobic and electrostatic interactions in nanomaterials. ${ }^{1,2}$ An essential further development in the application of supramolecular interactions is the step from nanoscale interactions to macroscopic materials. First examples using supramolecular architectures to induce a recognition between solid or soft materials were shown by Zhang et $a .^{3}{ }^{3}$ In that paper, multiple weak hydrogen bonding interactions were used to form stable hydrogels. In a recent example by Harada et al., hydrogels modified with guest molecules such as ferrocene, adamantane, azobenzene and alkyl chains show mesoscale recognition when mixed with cyclodextrin (CD) containing hydrogel blocks or surfaces. ${ }^{4-6}$ The interaction is based on hydrophobic effects between the cavity of the CD and the bulky hydrophobic residue. The size of the cavity (number of glucose units $\alpha=6, \beta=7$ and $\gamma=8$ ) determines the binding and the selectivity between the blocks. In contrast to Zhang et al., Ahn et al. developed a supramolecular velcro based on the reversible supramolecular interaction between ferrocene and cucurbit[7]uril (CB[7]). Complementary surfaces were functionalised

Organic Chemistry Institute and Center for Soft Nanoscience, Westfälische Wilhelms-Universität Münster, Corrensstrasse 40, 48149 Münster, Germany. E-mail:b.j.ravoo@uni-muenster.de

$\dagger$ Electronic supplementary information (ESI) available: Materials and methods, synthetic procedures, and additional spectra. See DOI: $10.1039 / \mathrm{c} 5 \mathrm{cc} 08968 \mathrm{a}$

\$ These authors contributed equally. with either $\mathrm{CB}[7]$ hosts or ferrocene guests. Upon contact of these surfaces, this glue held up to $4 \mathrm{~kg}$. Strong adhesion was attributed to the extraordinary affinity of the highly multivalent host-guest system. However, the supramolecular velcro was disrupted by oxidation since the resulting ferrocenium units do not bind to the $\mathrm{CB}$ cavity. ${ }^{7}$ Huskens et al. reported the selective immobilisation of silica nanoparticles on CD monolayers using adamantane functionalised dendrimers. ${ }^{8,9}$ In a more recent publication this concept was extended to different CD covered nanoparticles using redox active dendrimers, leading to a redox active supramolecular system. ${ }^{10}$ Several approaches to the fabrication of photoresponsive surfaces using azobenzenes have been described. ${ }^{11}$ Herges et al. showed efficient switching between the cis- and the trans-state in an azobenzene self-assembled monolayer (SAM). ${ }^{12,13}$ Zhang et al. described a switchable system based on the isomerisation of a fluorinated azobenzene SAM with increased wettability in the presence of $\alpha$-CD. Under UV-irradiation the CD is released and the hydrophobicity is recovered. ${ }^{14}$ Azobenzene units were also incorporated into surface grafted polymer brushes in order to yield photoresponsive polymer coatings. ${ }^{15-20}$ Our group used adamantane functionalised polymer brushes for the immobilisation of $\mathrm{CD}$ coated vesicles and silica nanoparticles mediated by host-guest interactions. ${ }^{21}$ We herein report on a novel approach for the preparation of a supramolecular adhesive based on surface grafted guest polymer brushes that have multiple interactions with a host polymer (Fig. 1). In earlier work we employed the reaction of triazolinedione (TAD) tagged molecules with cyclohexene functionalized surfaces in order to promote the layer-by-layer build-up of thin organic films. ${ }^{22}$ We also patterned 10-undecenyltrichlorosilane self-assembled monolayers (undecenyl SAMs) with a TAD tagged atom transfer radical polymerisation initiator (ATRP-TAD) to grow polyacrylate brushes in a graftingfrom approach. ${ }^{23}$ For this study, we adopt the latter strategy for the preparation of copolymer brushes consisting of 2-hydroxy ethyl acrylate (HEA) and an azobenzene substituted acrylate (AZA).

ATRP-TAD functionalised substrates were subjected to random copolymerisation using HEA and $7.5 \mathrm{~mol} \%$ of AZA in order to yield guest modified polymer brush surfaces. 


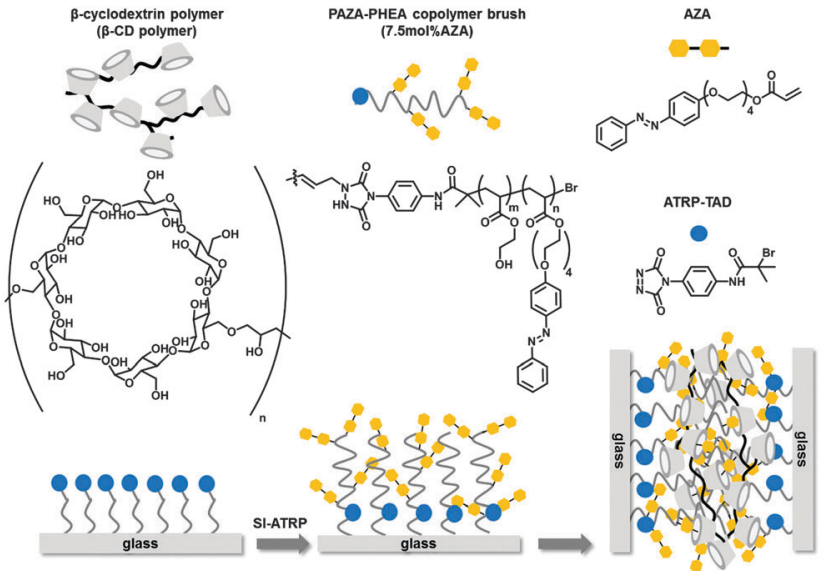

Fig. 1 Schematic representation of the stepwise construction of azobenzene containing PAZA-PHEA copolymer brushes, surface adhesion induced by the $C D$ polymer as well as the key molecular components used. $\mu C C$ : microcontact chemistry, ATRP: atom transfer radical polymerisation.

The brushes were investigated concerning their height and composition as well as their photophysical properties. In a first experiment a structured surface bearing line patterns of ATRPTAD was polymerised yielding well defined polymer brushes. These structured polymer brush substrates were used as references for convenient polymer brush height determination by atomic force microscopy (AFM). For all other experiments PAZA-PHEA copolymer brushes were grafted from surfaces which were fully functionalised with ATRP-TAD using flat non-patterned PDMS stamps. AFM showed a height of around $16 \mathrm{~nm}$ over a large area on the stripe patterns (Fig. 2a and ESI, $\dagger$ Fig. S2). To get a deeper insight into the consecutive steps of the surface modification, X-ray photoelectron spectroscopy (XPS) of the bare undecenyl SAM, the printed ATRP-initiator as well as grown PAZA-PHEA brushes was performed (Fig. 2b-d). The survey scan gives an overview of the whole scanned area (Fig. 2b). It is obvious that especially a
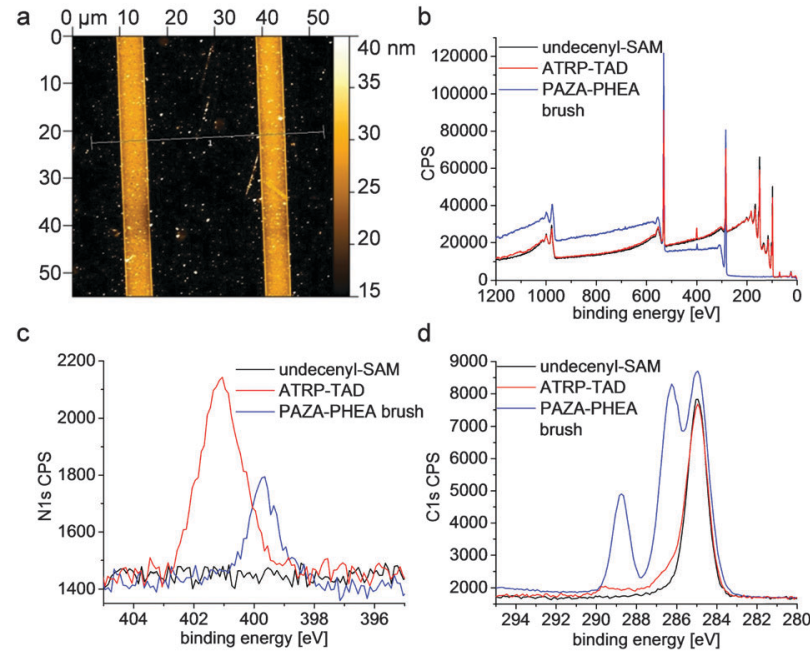

d

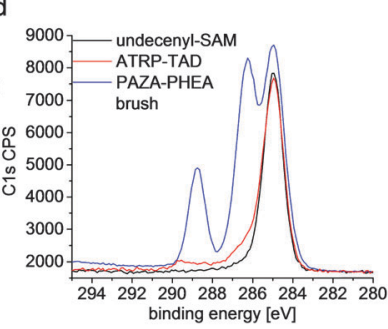

Fig. 2 (a) AFM-topography of PAZA-PHEA copolymer brushes structured in $5 \mu \mathrm{m}$ stripes which are spaced by $25 \mu \mathrm{m}$, (b) XPS survey scan, (c) XPS spectrum of the N1s-signal and (d) XPS spectrum of the C1s-signal of the alkene SAM, ATRP-TAD-SAM and PAZA-PHEA copolymer brushes. dramatic change in the range between 0 and $230 \mathrm{eV}$ is obtained when the polymer brushes are grown. This is easy to understand since these signals are due to silicon which is then shielded by the $16 \mathrm{~nm}$ polymer brushes. Taking a closer look at the nitrogen signal it is visible that after printing of the TAD initiator a characteristic signal at $c a .401 \mathrm{eV}$ occurs which belongs to the electron poor nitrogens in the heterocycle and the amide bond. (Fig. 2c) ${ }^{15}$ After polymerisation the signal vanishes due to shielding by the brushes and a new signal at lower binding energy (400 eV) occurs. This signal belongs to the less electron poor azobenzenes. Also the carbon signals showed the successful growth of the brushes (Fig. 2d). The alkene terminated SAM shows a single C1s signal at $285 \mathrm{eV}$, whereas the C1s peak of ATRP-TAD functionalised SAMs shows a small shoulder for higher oxidized carbons as well. After polymerisation, three different species stemming from the aliphatic backbone $(285 \mathrm{eV})$, higher oxidized carbons of the $\mathrm{C}-\mathrm{O}$ bonds contained in HEA side arms $(286 \mathrm{eV})$ and carboxylic carbons $(289 \mathrm{eV})$ are visible.

With these surfaces in stock the supramolecular adhesion ("supramolecular velcro") of homogeneously functionalized PAZAPHEA brush substrates was investigated. After incubation of one surface with the commercially available $\beta$-CD polymer $\left(100 \mathrm{mg} \mathrm{mL}^{-1}\right.$ in $\mathrm{H}_{2} \mathrm{O}$ ) for 10 min followed by washing with copious amounts of water a second surface was placed on top and the contacted substrates were dried for $1 \mathrm{~h}$ (area of contact $c a .1 .4 \mathrm{~cm} \times$ $1.3 \mathrm{~cm}$ ). Since azobenzenes are known to bind to $\beta-\mathrm{CD}$ we expected a strong interaction between the surfaces since a multivalent binding between azobenzene brushes and the $\beta$-CD polymer should occur. From Fig. 3a it is seen that the surfaces stick to each other. They were placed on a holder (Fig. $3 \mathrm{~b}$ ) and we attached weights in a step by step fashion in order to check for the maximum load ability of the supramolecular adhesive. As can be seen in Fig. 3c-e we reattached weights after every rupture of the surfaces to see if weakening of the sticking ability of the surfaces occurs. The disrupted surfaces were washed and sonicated with ethanol to remove the $\mathrm{CD}$ polymer, dried, reincubated with the $\beta$-CD polymer and finally placed on top of each other again. Upon the third reattachment, the strength of our system decreased to $50 \%$ as

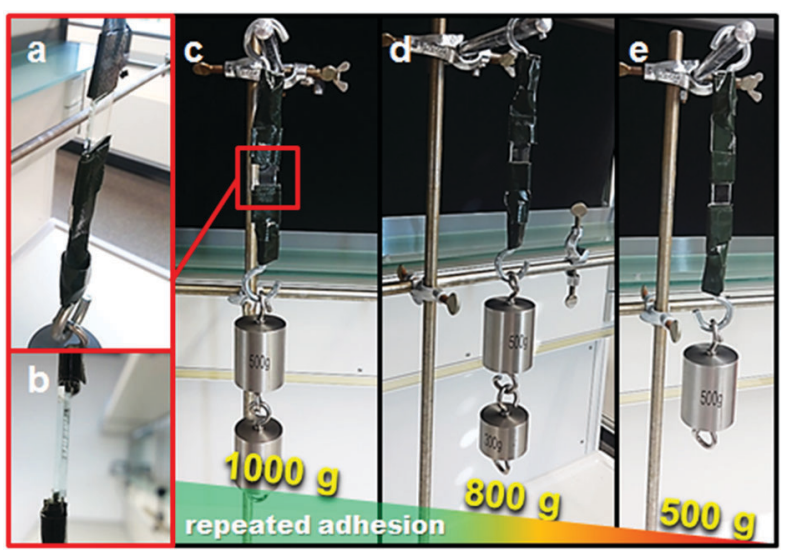

Fig. 3 ( $a$ and b) Close up photographs and setup of the glued glass surfaces, (c) weight attached, (d) weight attached after first rupture and (e) weight attached after second rupture. 

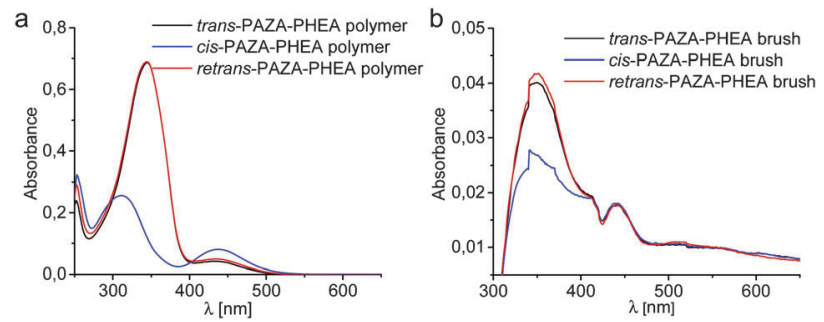

Fig. 4 UV/vis spectrum of (a) the bare PAZA-PHEA polymer grown in solution and (b) two glued PAZA-PHEA copolymer brush functionalised surfaces before and after irradiation with alternating $365 \mathrm{~nm}$ UV-light and visible light.

exemplified by Fig. 3e. The exact reasons for the weakening of adhesion strength remain elusive. However, weakening of adhesion may be due to surface contaminations or structural changes and deformations of polymer brushes. The average gluing capacity of the surfaces was determined to be $700 \pm 150 \mathrm{~g} \mathrm{~cm}^{-2}$. Control experiments without polymer brushes or without the $\beta$-CD polymer gave no significant interaction between the surfaces. The strength of the glue is related to the contact area and is increased to a value of $1.9 \mathrm{~kg}$ at a contact area of $2.24 \mathrm{~cm}^{2}$ (see the ESI, $\dagger$ Fig. S3).

Since it may be assumed that azobenzenes are involved in the binding process we expected a light-responsive behaviour of the brushes leading to the disruption of the surface adhesion under UV irradiation. Surprisingly, no significant effect of UV irradiation on the adhesion was observed. The lack of effect may have various reasons. On the one hand it is known that the photoirradiation of azobenzenes leads to a photostationary state, implying that even after extensive irradiation with UV light a significant amount of trans-azobenzene (ca. 20\%) remains so that multivalent binding persists. ${ }^{24-26}$ Furthermore the local concentration of the azobenzenes is high and the degree of translational and rotational freedom in the brushes is severely hindered, which could lead to an even less effective photo-isomerisation.

To get a deeper insight into the photophysical properties of the PAZA-PHEA copolymer brushes UV/vis measurements were conducted. To this end the AZA-monomer was measured in the trans-state and in the cis-state which is obtained after irradiation with UV light (365 nm) (see the ESI, $\dagger$ Fig. S1). A pronounced shift in absorption as well as the occurrence of a characteristic peak at $440 \mathrm{~nm}$ is observed. After irradiation with visible light (465 nm) the trans-form is re-obtained. The same spectral changes were also observed for the free polymer which is grown in solution during SI-ATRP at the surfaces (Fig. 4a). Thus, the polymer shows a similar behaviour to the free monomer indicating that the copolymerisation of HEA and AZA does not affect the photoisomerisation. In a further experiment two surfaces coated with PAZA-PHEA brushes were incubated with the CD polymer and placed on top of each other, following the aforementioned procedure. UV/vis measurements reveal a characteristic absorption at $360 \mathrm{~nm}\left(\pi \rightarrow \pi^{*}\right)$ (Fig. 4b). However, when irradiated with UV light at $365 \mathrm{~nm}$ a decrease in absorption was observed surprisingly without a shift to lower wavelength. Furthermore no increase of the absorption at $440 \mathrm{~nm}\left(\mathrm{n} \rightarrow \pi^{*}\right)$ was detectable. These findings lead to the assumption that a complete trans-cis isomerisation is not possible in the polymer brush and only a small amount of azobenzenes can undergo an isomerisation, explaining the stability of the glued surfaces under UV-light irradiation.

We present a supramolecular adhesive based on non-covalent host-guest interactions between azobenzenes in polymer brushes and a $\beta$-CD polymer. The brushes were investigated using AFM giving a height of $c a .16 \mathrm{~nm}$ and XPS verifying the presence of azobenzene in the brushes. Adhesion tests showed an average gluing capacity of around $700 \mathrm{~g} \mathrm{~cm}^{-2}$. Negative control experiments give no significant adhesion. As a consequence of high local concentration, multivalency, the unfavourable photostationary state and hindered rotational freedom, no light response of the supramolecular adhesion was observed. Nevertheless, this new type of polymer brush adhesive may find application in materials science as well as in surface related biomedical applications.

This work was funded by the Volkswagen Foundation.

\section{References}

1 C. Heinzmann, C. Weder and L. M. de Espinosa, Chem. Soc. Rev., 2015, DOI: 10.1039/C5CS00477B.

2 K. Autumn, Y. A. Liang, S. T. Hsieh, W. Zesch, W. P. Chan, T. W. Kenny, R. Fearing and R. J. Full, Nature, 2000, 405, 681-685.

3 R. Du, J. Wu, L. Chen, H. Huang, X. Zhang and J. Zhang, Small, 2014, 10, 1387-1393.

4 T. Nakamura, Y. Takashima, A. Hashidzume, H. Yamaguchi and A. Harada, Nat. Commun., 2014, 5, 4622.

5 Y. Takashima, T. Sahara, T. Sekine, T. Kakuta, M. Nakahata, M. Otsubo, Y. Kobayashi and A. Harada, Macromol. Rapid Commun., 2014, 35, 1646-1652.

6 T. Kakuta, Y. Takashima, T. Sano, T. Nakamura, Y. Kobayashi, H. Yamaguchi and A. Harada, Macromolecules, 2015, 48, 732-738.

7 Y. Ahn, Y. Jang, N. Selvapalam, G. Yun and K. Kim, Angew. Chem., Int. Ed., 2013, 52, 3140-3144.

8 V. Mahalingam, S. Onclin, M. Péter, B. J. Ravoo, J. Huskens and D. N. Reinhoudt, Langmuir, 2004, 20, 11756-11762.

9 O. Crespo-Biel, B. Dordi, P. Maury, M. Péter, D. N. Reinhoudt and J. Huskens, Chem. Mater., 2006, 18, 2545-2551.

10 X. Y. Ling, D. N. Reinhoudt and J. Huskens, Chem. Mater., 2008, 20, 3574-3578.

11 R. Klajn, Pure Appl. Chem., 2010, 82, 2247-2279.

12 U. Jung, O. Filinova, S. Kuhn, D. Zargarani, C. Bornholdt, R. Herges and O. Magnussen, Langmuir, 2010, 26, 13913-13923.

13 U. Jung, C. Schütt, O. Filinova, J. Kubitschke, R. Herges and O. Magnussen, J. Phys. Chem. C, 2012, 116, 25943-25948.

14 P. Wan, Y. Jiang, Y. Wang, Z. Wang and X. Zhang, Chem. Commun., 2008, 5710-5712.

15 H. A. Haque, S. Kakehi, M. Hara, S. Nagano and T. Seki, Langmuir, 2013, 29, 7571-7575.

16 H. A. Haque, S. Nagano and T. Seki, Macromolecules, 2012, 45, 6095-6103. 17 D. Wang, G. Ye, X. Wang and X. Wang, Adv. Mater., 2011, 23, 1122-1125.

18 A. Dirani, X. Laloyaux, A. E. Fernandes, B. Mathy, O. Schicke, O. Riant, B. Nysten and A. M. Jonas, Macromolecules, 2012, 45, 9400-9408.

19 C. Schuh, N. Lomadze, J. Rühe, A. Kopyshev and S. Santer, J. Phys. Chem. B, 2011, 115, 10431-10438.

20 N. Lomadze, A. Kopyshev, J. Rühe and S. Santer, Macromolecules, 2011, 44, 7372-7377.

21 O. Roling, A. Mardyukov, J. A. Krings, A. Studer and B. J. Ravoo, Macromolecules, 2014, 47, 2411-2419.

22 B. Vonhören, O. Roling, K. De Bruycker, R. Calvo, F. E. Du Prez and B. J. Ravoo, ACS Macro Lett., 2015, 4, 331-334.

23 O. Roling, K. De Bruycker, B. Vonhören, L. Stricker, M. Körsgen, H. F. Arlinghaus, B. J. Ravoo and F. E. Du Prez, Angew. Chem., Int. Ed., 2015, 54, 13126-13129.

24 S. K. M. Nalluri, J. Voskuhl, J. B. Bultema, E. J. Boekema and B. J. Ravoo, Angew. Chem., Int. Ed., 2011, 50, 9747-9751.

25 C. Stoffelen, J. Voskuhl, P. Jonkheijm and J. Huskens, Angew. Chem., Int. Ed., 2014, 53, 3400-3404.

26 J. Voskuhl, S. Sankaran and P. Jonkheijm, Chem. Commun., 2014, 50, 15144-15147. 